\title{
Efficacy Analysis of Glucocorticoids in the Treatment of Allergic Purpura in Tibetan Children
}

\author{
Xiangyu Zhang ${ }^{*}$, Zhaxi Nima ${ }^{1 *}$, Shunde Zhang ${ }^{*}$, Wangdui Suona ${ }^{1}$, Ciren Pubu${ }^{1}$, Shengyou Yu ${ }^{2 \#}$ \\ ${ }^{1}$ Department of Pediatrics, People's Hospital of BoMi County, Tibet Autonomous Region, China \\ ${ }^{2}$ Department of Pediatrics, Guangzhou First People's Hospital, School of Medicine, South China University of Technology, \\ Guangzhou, China \\ Email: "shengyouyu@yeah.net
}

How to cite this paper: Zhang, X.Y., Nima, Z., Zhang, S.D., Suona, W., Pubu, C. and Yu, S.Y. (2021) Efficacy Analysis of Glucocorticoids in the Treatment of Allergic Purpura in Tibetan Children. International Journal of Clinical Medicine, 12, 14-22. https://doi.org/10.4236/ijcm.2021.121003

Received: November 30, 2020

Accepted: January 11, 2021

Published: January 14, 2021

Copyright $\odot 2021$ by author(s) and Scientific Research Publishing Inc. This work is licensed under the Creative Commons Attribution International License (CC BY 4.0).

http://creativecommons.org/licenses/by/4.0/

\begin{abstract}
Background: Henoch Schonlein Purpura (HSP) is a common immune-related allergic disease in children. It is very important to understand the clinical features of this disease for doctors. Objective: To explore the clinical efficacy of glucocorticoids in the treatment of HSP in Tibetan children at high altitude, and to analyze the possible causes of HSP in children at high altitude. The risk factors of the disease provide a reference for the treatment of HSP in children in high altitude areas. Methods: Selecting January 2015 to November 2020, 88 children diagnosed with Henoch-Schonlein Purpura in the outpatient and inpatient departments of the People's Hospital of Bomi County, Tibet Autonomous Region were the subjects of the study. Its gender, age of onset, season of onset, predisposing factors, allergy history, first symptoms, clinical manifestations, laboratory examinations, etc., perform retrospective analysis. Results: Among 88 children with allergic purpura, 55 were boys, accounting for $62.5 \%$, and 33 were girls, accounting for $37.5 \%$. Men have more cases than women. All have clinical manifestations of purpura of the skin, among which 35 cases have obvious triggers, of which the above there were 26 cases of respiratory infections, 6 cases of dietary factors, and 3 cases of contact with allergic substances. Simple skin type: 18 cases, accounting for 20.45\%; Abdominal type: 6 cases, accounting for $6.82 \%$; 2 male cases, accounting for $33.33 \%$; 4 female cases, accounting for $66.67 \%$; Articular type: 8 cases, accounting for 33.33\%; Renal type: 2 cases, accounting for $2.27 \%$; 54 cases of mixed type, accounting for $61.36 \%$. After glucocorticoids, the rashes disappeared, no any adverse reactions. Conclusion: Allergic purpura in children
\end{abstract}

${ }^{\star}$ Zhang-Xiangyu, Nima Zhaxi and Zhang-Shunde contributed equally to this work. They are the co-first authors. 
is more common in school-age children, and upper respiratory tract infection is the main predisposing factor. Skin purpura is the main clinical manifestation, often associated with lower extremity joint swelling and pain. There is no significant difference in the efficacy and course of the disease between intravenous and oral treatment. Therefore, clinicians should strictly grasp the indications of glucocorticoids to reduce the occurrence of complications.

\section{Keywords}

Plateau, Tibetan, Children, Glucocorticoids, Allergic Purpura

\section{Introduction}

HSP is a systemic vasculitic disease which is common in children, and one of the systemic vasculitis, mainly with capillary vasculitis as the main pathological change, and its pathological change is extensive aseptic capillitis. It is accompanied by edema and congestion [1] [2]. The non-thrombocytopenic purpura, arthritis or joint pain, abdominal pain, gastrointestinal bleeding and nephritis are the main clinical manifestations. The common age of onset is $7-14$ years old, and it is rare in infants under 1 year-old [3] [4]. Its etiology and pathogenesis are complex. The mechanism is not clear, and the incidence of the disease has been increasing year by year in recent years. At present, the clinical treatment methods are still mainly anti-allergic and symptomatic treatment. But severe cases are often accompanied by gastrointestinal tract, and kidneys injuries and other organ injuries [5] [6]. It requires clinicians to adopt appropriate diagnosis and treatment plans in time. Current research mostly focuses on plains. The treatment plan and clinical characteristics of children with HSP are the main ones. There is still a lack of relevant data on the treatment of HSP in children with high altitude Tibetans, and there is insufficient reference for clinical diagnosis and treatment. This study focuses on retrospective analysis of the 88 cases of HSP children treated in the Department of Pediatrics of the People's Hospital of Bomi County, Tibet Autonomous Region from January 2015 to November 2020, and provides recommendations for the treatment of HSP in the plateau area.

\section{Clinical Data and Methods}

\subsection{Inclusion Criteria [7]}

1) Age 1 - 16 years old, native Tibetan children of plateau; 2) Meet the diagnostic criteria of HSP; 3) Do not combine with other types of purpura (such as blood Small plate reduced purpura) and other blood system diseases (such as leukemia); 4) The clinical data is perfect. 


\subsection{Exclusion Criteria [7]}

1) Congenital dysplasia, hepatosplenomegaly, systemic lupus erythematosus; 2) Recent severe systemic infection, primary kidney disease, such as kidney Glomerulonephritis; 3) Recently took glucocorticoids or other immunosuppressive agents.

\subsection{Treatment Methods}

All 88 children with Henoch-Schonlein purpura were treated with glucocorticoids, antihistamines, vitamin $\mathrm{C}$ and calcium, and their condition improved. Children were given oral or intravenously injected with dexamethasone (Huanan Pharmaceutical Group Co., Ltd., Guangdong, China, State Drug Approval Document Number: H44024469) at $0.25 \mathrm{mg} /(\mathrm{kg} \cdot \mathrm{d})$. The drug was gradually reduced to stop.

\subsection{Efficacy Criteria}

Healed: Skin purpura subsides, and other symptoms disappear; Significant effect: Most skin purpura subsides, and symptoms are significantly reduced; Effective: Skin purpura partially subsides, and the symptoms have improved; ineffective: skin purpura recurs, and other symptoms aggravate.

\subsection{Statistical Processing}

Application SPSS 25.0 software for processing. Counting data is expressed as a percentage, measurement data is expressed as $\bar{x} \pm \mathrm{s}, \mathrm{P}<0.05$ is statistically significant.

\section{Results}

\subsection{General Situation}

Among 88 cases of Henoch-Schonlein Purpura, 55 were boys, accounting for $62.5 \%$, and girls: 33 , accounting for $37.5 \%$. Men had more cases than women. The results are shown in Table 1 . The age of onset is 1 to 16 years old, with 1 infant, accounting for $1.14 \%$; 22 cases from 5 to 7 years old, accounting for $25 \%$; 44 cases were 8 to 12 years old, accounting for 50\%; 21 cases were 13 to 16 years old, accounting for $23.86 \%$. The results are shown in Table 2.

\subsection{Clinical Feature}

Among the 88 children with Henoch-Schonlein purpura, the simple skin type: 18 cases, accounting for $20.45 \%$, including 15 males, accounting for $83.33 \%$, females 3 cases, accounting for 16.67\%; abdominal type: 6 cases, accounting for $6.82 \%, 2$ male cases, accounting for $33.33 \%, 4$ female cases, accounting for $66.67 \%$; 8 cases of articular type, accounting for $9.1 \%$, of which 4 were male, accounting for $50 \%$, and 4 were female, accounting for 50\%; renal type: 2 cases, accounting for $2.27 \%$, and There are 2 males, accounting for $100 \%, 0$ females, 
accounting for 0\%; 54 mixed cases, accounting for $61.36 \%$, of which 32 are males, accounting for $59.26 \%, 22$ cases were female, accounting for $40.74 \%$. The results are shown in Table 3.

\subsection{Performance of Skin Damage}

All 88 children with allergic purpura had clinical manifestations of skin purpura, among which 35 cases had obvious triggers, among which the upper respiratory tract infection. There were 26 cases of infection, 6 cases of dietary factors, and 3 cases of contact with allergic substances. From the point of view of the distribution of purpura, most of them involve double under. The limbs are distributed symmetrically, followed by the hips and upper limbs. The back of hands, feet, trunk and face are rare. It may be accompanied by a sensation of itching. In severe cases, it may be accompanied by angioedema, purpura or hemorrhagic maculopapular rash of various sizes, different shapes, and pressure. The color does not fade, and sometimes it can be fused into a piece. The time for the rash to resolve varies depending on the condition of the disease, mostly ranging from $5 \mathrm{~d}$ 7 days (Figure 1 ).

\subsection{Treatment Time}

88 cases of children with Henoch-Schonlein purpura according to the course of the disease: 60 cases within 10 days of treatment, 26 cases within 10 - 15 days, and 2 cases were more than 15 days. For example, the results are shown in Table 4. Among them, 36 children were treated with intravenous glucocorticoids, 29 cases were treated for $\leq 10$ days, and 10 - 15 days accounted for 5 cases, 2 cases were more than 15 days or more, the results are shown in Table 5.52 children were treated with oral corticosteroids, and the treatment was less than 10 days, Among 31 cases, 10 - 15 days accounted for 21 cases, and $\geq 15$ days 0 cases. The results are shown in Table 6.

Table 1. Sex of 88 cases of Henoch-Schonlein purpura.

\begin{tabular}{ccc}
\hline Gender & Number of cases & Percentage (\%) \\
\hline Male & 55 & 62.5 \\
Femal & 33 & 37.5
\end{tabular}

Table 2. Age of onset of 88 cases of Henoch Schonlein purpura.

\begin{tabular}{ccc}
\hline Age & Number of cases & Percentage (\%) \\
\hline Infancy $(<1$ years old $)$ & 1 & 1.14 \\
Preschool $(5-7$ years old $)$ & 22 & 25 \\
School age $(8-12$ years old $)$ & 44 & 50 \\
Puberty $(13-16$ years old $)$ & 21 & 23.86
\end{tabular}


Table 3. Treatment time of 88 cases of Henoch-Schonlein purpura.

\begin{tabular}{ccc}
\hline Days & Number of cases & Percentage (\%) \\
\hline Simple skin type & 18 & 20.45 \\
Abdominal type & 6 & 6.82 \\
Articular type & 8 & 9.1 \\
Renal type & 2 & 2.27 \\
Mixed type & 54 & 61.36 \\
\hline
\end{tabular}

Table 4.88 cases of allergic purpura treatment time.

\begin{tabular}{ccc}
\hline Days & Number of cases & Percentage (\%) \\
\hline 10 days & 60 & 68.2 \\
$10-15$ days & 26 & 29.5 \\
$\geq 15$ days & 2 & 2.3 \\
\hline
\end{tabular}

Table 5. 36 cases of intravenous glucocorticoid treatment time.

\begin{tabular}{ccc}
\hline Days & Number of cases & Percentage (\%) \\
\hline$\leq 10$ days & 29 & 80.6 \\
$10-15$ days & 5 & 13.9 \\
$\geq 15$ days & 2 & 5.5 \\
\hline
\end{tabular}

Table 6. Treatment time of 52 cases of oral corticosteroids.

\begin{tabular}{ccc}
\hline Days & Number of cases & Percentage (\%) \\
$\leq 10$ days & 31 & 59.6 \\
$10-15$ days & 21 & 40.4 \\
$\geq 15$ days & 0 & 0
\end{tabular}

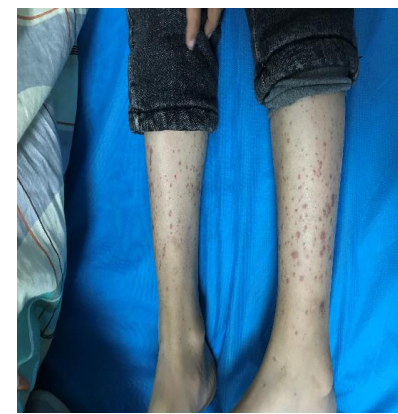

Before treatment

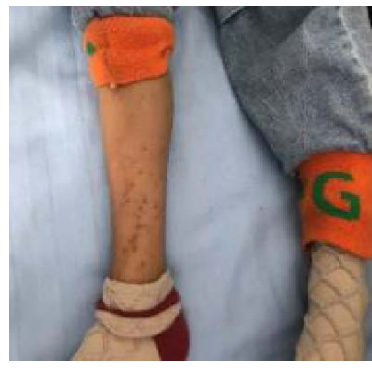

Before treatment

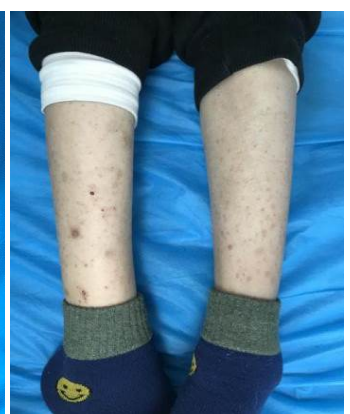

After treatment

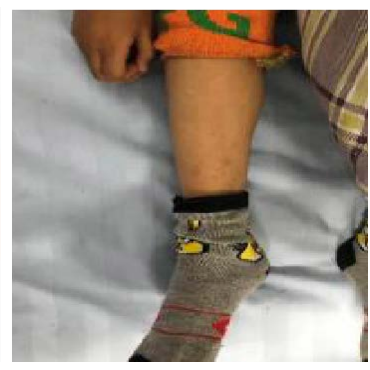

After treatment

Figure 1. After treatment, the child's rash subsided. 


\section{Discussion}

In recent years, the prevalence of allergic purpura in children has been increasing. The pathogenesis is mainly immunological abnormalities and humoral immune abnormalities. It also involves cellular immunity with the participation of inflammatory cells, cytokines and inflammatory mediators. There are microvascular and interstitial in renal pathology damage. Non-thrombocytopenia and palpable skin purpura are necessary conditions for the diagnosis of HSP, and the rash is not the only phenotype in all children. Some children have joint pain or abdominal pain as the first symptoms, which can be as long as two weeks without rash [8]. Therefore, it is often easy to cause misdiagnosis. For example, when some children often have lower limbs or ankle joint swelling and painas the first symptom, parents think it is caused by falls or sprains [9], or some children have purpura nephritis. When symptoms such as hypertensive encephalopathy will occur, if treatment is delayed [10] [11]; even when HSP abdominal type has not yet appeared skin purpura or has appeared but has not been detected, it is misdiagnosed as acute For acute abdomen such as appendicitis, laparotomy is performed. The symptoms of abdominal pain in children cannot be relieved or further aggravated, causing a series of complicationsand increasing the difficulty of follow-up treatment [12]. Therefore, clinicians must be vigilant for children with unexplained abdominal pain before the appearance of typical skin purpura, provide auxiliary examinations if necessary, and be cautious in laparotomy to avoid misdiagnosis that may increase the pain and damage the health of the child.

At present, there is no specific treatment plan for HSP, and comprehensive treatment is still the mainstay. Adrenal corticosteroids are added to those with severe gastrointestinal symptoms and joint swelling and pain, and those with nephropathy or rapidly progressive nephritis are treated with methylprednisolone shock treatment [13] [14]. For HSP treatment, it is necessary to strictly grasp the indications, to improve the clinical prognosis of children to the greatest extent, and to actively give immunosuppressive treatment and support symptomatic treatment [15]. In addition, the study also found that some children have kidney damage, and the degree of kidney damage is critical to the prognosis. The increase in urine microprotein excretion can be earlier than urine routine or urine protein quantitative examination, which is helpful for the early diagnosis of occult kidney damage [16] [17]. This study shows that among 88 allergic purpura cases, 55 are boys, accounting for $62.5 \%$, and 33 are girls, accounting for $37.5 \%$ of men have more cases than women. The age of onset ranges from 1 to 17 years old. There is 1 case of infants and young children, accounting for 1.14\%; 5 to 7 years old 22 cases were aged 8 to 12 years, accounting for $25 \%$; 44 cases were 8 to 12 years old, accounting for $50 \%$; 21 cases were 11 to 16 years old, accounting for $23.86 \%$. Among them, the simple skin type: 18 cases, accounting for $20.45 \%$, of which 15 cases are male, accounting for $83.33 \%$, and 3 cases are female, accounting for 16.67\%; Abdominal type: 6 cases, accounting for $6.82 \%, 2$ male cases, accounting for $33.33 \%, 4$ female cases, accounting for 66.67\%; articular type 8 cases, accounting for $33.33 \%$, including 4 males, ac- 
counting for 50\%, 4 females, accounting for 50\%; kidney type: 2 cases, accounting for $2.27 \%$, including 2 males, accounting for $100 \%$, 0 females, accounting for $0 \%$; 54 mixed cases, accounting for $61.36 \%$, including 32 males, accounting for $59.26 \%$, females 22 cases, accounting for $40.74 \%$. All children have clinical manifestations of skin purpura, of which 35 cases have obvious triggers, of which the above. There were 26 cases of respiratory infections, 6 cases of dietary factors, and 3 cases of contact with allergic substances. From the distribution of purpura, it affects both lower extremities and is symmetrically distributed. Secondly, it can affect the buttocks and upper extremities. The back of hands, feet, trunk and face are less common. Sometimes purpura may also be accompanied by itching, in severe cases it may be accompanied by angioedema, purpura or hemorrhagic maculopapular rashes of various sizes and shapes. It is different and does not fade under pressure. Sometimes it can be fused into a piece. The time for the rash to subside varies with the treatment of the condition, mostly ranging from 5d - 7 days. Among them, 60 cases were treated within 10 days, 26 cases were treated for 10 - 15 days, and 2 cases were more than 15 days. Among them, 36 children were static. Glucocorticoid treatment, 29 cases were treated for $\leq 10$ days, 5 cases were 10 - 15 days, 2 cases were $\geq 15$ days, 52 cases were children with oral glucocorticoid therapy, 31 cases were treated within 10 days, and 21 cases were treated within 10 - 15 days, confirmed that there was no difference between intravenous and oral corticosteroids in the curative effect and course.

\section{Conclusion}

In summary, Henoch-Schonlein Purpura is a common and frequently-occurring disease in children, and can involve multiple systemic systems. Factors are related. The age of onset of allergic purpura in our region is mostly $8-12$ years old, male > female; mixed purpura is common; simple skin is second; most of them have no cause for onset; there is no significant difference in curative effect and course of disease between intravenous and oral treatment, so clinicians should strictly grasp the indications of glucocorticoids to reduce the occurrence of complications. Due to the small number of observations and short time in this study, its overall long-term efficacy needs to be further confirmed in a multi-center, large sample, prospective clinical double-blind randomized controlled study.

\section{Acknowledgements}

This study was supported by the Science Foundation of Guangzhou First People's Hospital (No.M2019020); Guangdong Medical Science and Research Foundation (No.A2018539); Guangzhou General Science and Technology Project of Health and Family Planning (No.20181A011004).

\section{Conflicts of Interest}

The authors declare no conflicts of interest regarding the publication of this paper. 


\section{References}

[1] Wilkinson, A. (2019) Early Recognition and Treatment of Henoch-Schönlein Purpura in Children. Nursing Children and Young People, 31, 36-40.

https://doi.org/10.7748/ncyp.2019.e1118

[2] Karadağ, Ş.G., Tanatar, A., Sonmez, H.E., Çakmak, F., Kıyak, A., Yavuz, S., Çakan, M. and Ayaz, N.A. (2019) The Clinical Spectrum of Henoch-Schönlein Purpura in Children: A Single-Center Study. Clinical Rheumatology, 38, 1707-1714. https://doi.org/10.1007/s10067-019-04460-1

[3] Ekinci, R.M.K., Balci, S., Melek, E., Karabay Bayazit, A., Dogruel, D., Altintas, D.U. and Yilmaz, M. (2020) Clinical Manifestations and Outcomes of 420 Children with Henoch Schönlein Purpura from a Single Referral Center from Turkey: A Three-Year Experience. Modern Rheumatology, 30, 1039-1046. https://doi.org/10.1080/14397595.2019.1687074

[4] Gómez, S., Pérez, M., Pellegrini, M., Isern, E., Quintana, C., Artacho, P., Bertolini, M., Pomerantz, B. and Gadda, N. (2020) Henoch-Schonlein Purpura in Pediatrics: Ten Years of Experience at a Moderate Risk Office of a General Hospital. Archivos Argentinos de Pediatria, 118, 31-37. https://doi.org/10.5546/aap.2020.eng.31

[5] Szemenyei, C. and Hahn, D. (2015) Prevention of Nephritis in Henoch-Schönlein Purpura. Journal of Paediatrics and Child Health, 51, 236-239. https://doi.org/10.1111/jpc.12785

[6] Çakıcı, E.K., Gür, G., Yazılıtaş, F., et al. (2019) A Retrospective Analysis of Children with Henoch-Schönlein Purpura and Re-Evaluation of Renal Pathologies Using Oxford Classification. Clinical and Experimental Nephrology, 23, 939-947. https://doi.org/10.1007/s10157-019-01726-5

[7] Zeng Chuanwen, Gesang Deji, Dawa Quzhen, et al. (2019) Analysis of Related Risk Factors for Allergic Purpura in Tibetan Children on Plateau. Chinese Critical Care Medicine, 31, 742-745.

[8] Wang, H., Das, L., Hoh, S.F., et al. (2019) Urinalysis Monitoring in Children with Henoch-Schönlein Purpura: Is It Time to Revise? International Journal of Rheumatic Diseases, 21, 145-147.

[9] Tan, J., Tang, Y., Zhong, Z., et al. (2019) The Efficacy and Safety of Immunosuppressive Agents plus Steroids Compared with Steroids Alone in the Treatment of Henoch-Schönlein Purpura Nephritis: A Meta-Analysis. International Urology and Nephrology, 51, 975-985. https://doi.org/10.1007/s11255-019-02092-7

[10] Thong, P.L., How, K.N. and Bakrin, I.H. (2020) Hypertension in the Absence of Urinary Abnormalities-An Unusual Presentation of Anaphylactoid Purpura. Medical Journal of Malaysia, 75, 436-438.

[11] Da Dalt, L., Zerbinati, C., Strafella, M.S., et al. (2016) Henoch-Schönlein Purpura and Drug and Vaccine Use in Childhood: A Case-Control Study. Italian Journal of Pediatrics, 42, Article No. 60. https://doi.org/10.1186/s13052-016-0267-2

[12] Albaramki, J. (2016) Henoch-Schönlein Purpura in Childhood a Fifteen-Year Experience at a Tertiary Hospital. Le Journal Medical Libanais, 64, 13-17. https://doi.org/10.12816/0023826

[13] Yu, S.Y., Yu, L. and Wang, L.N. (2011) Clinical Efficacy of Methylprednisolone Shock-Decreasing Therapy in the Treatment of Severe Allergic Purpura. Practical Journal of Clinical Pediatrics, 26, 696-698.

[14] Buscatti, I.M., Casella, B.B., Aikawa, N.E., et al. (2018) Henoch-Schönlein Purpura Nephritis: Initial Risk Factors and Outcomes in a Latin American Tertiary Center. 
Clinical Rheumatology, 37, 1319-1324. https://doi.org/10.1007/s10067-017-3972-3

[15] Hetland, L.E., Susrud, K.S., Lindahl, K.H., et al. (2017) Henoch-Schönlein Purpura: A Literature Review. Acta Dermato-Venereologica, 97, 1160-1166.

https://doi.org/10.2340/00015555-2733

[16] Delbet, J.D., Hogan, J., Aoun, B., et al. (2017) Clinical Outcomes in Children with Henoch-Schönlein Purpura Nephritis without Crescents. Pediatric Nephrology, 32, 1193-1199. https://doi.org/10.1007/s00467-017-3604-9

[17] Hahn, D., Hodson, E.M., Willis, N.S. and Craig, J.C. (2015) Interventions for Preventing and Treating Kidney Disease in Henoch-Schönlein Purpura (HSP). Cochrane Database of Systematic Reviews, 8, CD005128. https://doi.org/10.1002/14651858.CD005128.pub3 